\title{
Functional treatment in rotator cuff tears: is it safe and effective? A retrospective comparison with surgical treatment
}

\author{
Angelo De Carli ${ }^{1}$ \\ Mattia Fabbri ${ }^{1}$ \\ Riccardo Maria Lanzetti ${ }^{1}$ \\ Alessandro Ciompi ${ }^{1}$ \\ Edoardo Gaj ${ }^{1}$ \\ Gioia Beccarini ${ }^{1}$ \\ Mario Vetrano² \\ Andrea Ferretti ${ }^{1}$ \\ 1 Orthopaedic Unit and "Kirk Kilgour" Sports Injury \\ Centre, Sant'Andrea Hospital, "Sapienza" Universi- \\ ty of Rome, Rome, Italy \\ 2 Physical Medicine and Rehabilitation Unit, Sant'An- \\ drea Hospital, "Sapienza" University of Rome, \\ Rome, Italy
}

Corresponding author:

Mattia Fabbri

Orthopaedic Unit and "Kirk Kilgour" Sports Injury

Centre, Sant'Andrea Hospital, "Sapienza"

University of Rome

Via di Grottarossa 1035

00189 Rome, Italy

E-mail: docmattiafabbri@gmail.com

\section{Summary}

Background: The aim of this study was to compare rehabilitation protocol and operative treatment in a population of patients with a diagnosis of small to medium rotator cuff tears $(\leq 3 \mathrm{~cm})$, the null hypothesis being that there would been no difference in terms of clinical outcomes and patient's satisfaction between the rehabilitation protocol and the surgical treatment.

Methods: Patients with small to medium supraspinatus tears were retrospectively enrolled in this study and divided in 2 groups: arthroscopic repair (group A, 20 patients) and reinstated (group B, 18 patients). At a mean follow-up of 18 months, both groups underwent clinical (Constant, QuickDash, VAS), dynamometric and ultrasonographic evaluation.

Results: In both groups a significant clinical improvement was registered compared to baseline. However, surgical treatment yielded better results in Constant $(p=0.004)$, Quick-Dash $(p=0.0012)$, VAS $(p=0.048)$ and strength evaluation $(p=0.0014)$. In group $A$ the re-tear rate was $10 \%$, while in group $B$ only $11 \%$ of increased tear size was registered. Conclusion: At a short term follow-up, the surgical treatment of small to medium supraspinatus tears yielded better clinical outcomes compared to the rehabilitation protocol, with better strength outcomes and $10 \%$ re-tear rate. Nevertheless, physiotherapy still offers acceptable results and could be a valuable option in patients not undergoing surgery.

Level of evidence: III.

KEY WORDS: rehabilitation protocol, functional treatment, rotator cuff tear, surgery, strength.

\section{Introduction}

Rotator cuff tears (RCT) are one of the most frequent shoulder diseases and their incidence increases with advancing age 1 . Nevertheless, only $1 / 3$ of the tears come to observation, which means that most tears are painless with only little functional limitation ${ }^{2}$. Since the first report by Codman ${ }^{3}$, several arthroscopic and mini-open techniques have been proposed for the repair of such lesion, with different results ${ }^{4-8}$. Surgical repair is performed in patients with reparable symptomatic lesions with a high functional demand of the shoulder. The Rehabilitation protocol included active surveillance, shoulder exercises, and oral pain medication if necessary.

Conservative physical therapies have been reported to have acceptable results by many Authors ${ }^{9-14}$. Kukkonen et al. in 2014 illustrated that conservative treatment should be considered as the primary method of treatment for non-traumatic rotator cuff tears ${ }^{15}$. Thus surgical indication must be tailored to each patient considering psychological factors, expectations and the presence of systemic disease contraindicating surgery. Therefore, the aim of this study is to compare the rehabilitation protocol and the surgical treatment in a population of patients with a diagnosis of small to medium $(\leq 3 \mathrm{~cm})$ reparable rotator cuff tears ${ }^{16}$, the null hypothesis being that there would been no difference in terms of clinical outcomes and patient's satisfaction between the rehabilitation protocol and the surgical treatment.

\section{Materials and methods}

We retrospectively identified from our clinical database 75 patients with clinical and radiological evi- 
dence of RCT who arrived at the first orthopaedic visit between 6 months and 2 years after the appearance of symptoms. The subjects had been recruited between January 2012 and December 2014. Patients were homogeneous for work activity (no case of heavy work). Inclusion criteria were: small to mediumsize $(\leq 3 \mathrm{~cm})$ according to Cofield $\mathrm{RH}^{16}$, symptomatic full thickness supraspinatus tears; age between 50 and 75 years; indication for surgical repair. Exclusion criteria were considered: previous shoulder surgery; physical therapy before orthopaedic examination; contralateral rotator cuff repair; bilateral involvement as confirmed by ultrasound in symptomatic patients; multiple tendon involvement; frozen shoulder; rheumatoid arthritis; neurological disorders; radiologic and symptomatic osteoarthritis of the glenohumeral or acromioclavicular joint.

35 patients, which did not meet inclusion criteria, were excluded, leaving 40 consecutive patients giving their consent to be included in this study: 20 patients were treated with surgical repair (group A), while 20 underwent conservative treatment (group B). The reasons for selecting conservative treatment were concomitant disorders contraindicating surgery in 12 patients (pulmonary disease, 4 patients; previous cardiac disease, 7 patients; and unstable angina pectoris, 1 patients) $(66.7 \%)$ and personal reasons in 8 patients (33.3\%).

Patients with diabetes or hormonal diseases were absent in both groups. Hypertension and hypercholesterolemia were equally distributed in both groups.

Group A underwent the same surgical technique: arthroscopic repair was performed with patient in lateral decubitus. Standard arthroscopic portals were used. An arthroscopic single-row repair was performed in all the cases with non absorbable suture anchor (Corkscrew, Arthrex $\left.{ }^{\circledR}\right)$. Acromioplasty was performed only in patients in which signs of subacromial post-suture impingement were arthroscopically detected. When a long head biceps pathology was found, a tenotomy/tenodesis or a tenotomy alone was performed according to patient's age ${ }^{17}$. After surgery all patients underwent the same rehabilitation protocol: 4 weeks wearing a brace with shoulder in neutral position, starting passive exercises at week 2 . From week 5 , assisted passive and active exercises in order to regain range of motion (ROM) and strength were gradually started. The complete recovery of functional activity was allowed after three months.

Group B underwent a standardized rehabilitation protocol structured in 3 weekly sessions separated by a day of rest, for a total of 12 sessions performed with the help of the same physiotherapist. After this period exercises were performed on a home-based protocol by patients with the same frequency and similar exercises. The treatment consisted of passive, assisted active and active exercises; initial evaluation of the scapular position compared to the contralateral one; scapular detachment; simple passive mobilization in flexion, abduction, external rotation, associated with optional movements at end of ROM; bilateral stretching of the pectoralis minor; active mobilization ac- cording to Kabat's ${ }^{18}$ scheme in flexion, adduction and external rotation with the elbow extended and in flexion, abduction and external rotation with elbow extended; cognitive therapeutic exercise (CTE) performed through the identification of different pressures on the scapula and the identification of different circular paths. Physical therapy had not been administered in both the groups of patients. Patients of both groups were educated to the assumption of NSAIDs (paracetamol) as needed.

At follow-up, patients were required to express degree of personal compliance to the protocol with a scale ranging from 1 to 3 (1: poor compliance; 2: mean compliance; 3 : full compliance).

All patients enrolled underwent a standardized clinical evaluation. In group $A$, this was conducted at the time of diagnosis (T0) and at the latest follow-up (T2, $18 \pm 2$ months). In group $B$, it was conducted at the time of diagnosis (TO), at the intermediate follow-up (T1, $6 \pm 1$ months) and at the latest follow-up (T2, $18 \pm 3$ months). Patients were asked to complete Constant Score ${ }^{19}$, QuickDash ${ }^{20}$ questionnaires and Visual Analogic Scale ${ }^{21}$ (VAS) in order to assess the shoulder function. Moreover, patient's satisfaction at T2 was investigated with a scale ranging from 1 to 4 (1: Unsatisfied; 2: Poor satisfaction; 3: Good satisfaction; 4: Excellent satisfaction).

At the latest follow-up (T2) patients from both groups underwent a standardized strength assessment using a digital dynamometer (Portable dynamometer HDD1-500 Kgp, Lbt, N). Arm abduction at $45^{\circ}$ was performed with the elbow fully extended and internal rotation (empty can) on the scapular plane. Two trials on each upper limb were undertaken, alternating between the involved and the uninvolved limbs, with the first trial always undertaken on the uninvolved side. During each trial, patients were asked to perform to their maximum muscle strength. Patients were given adequate rest between trials to minimize fatigue. Strength values were recorded as Kiloponds and reported as percentage of involved/uninvolved limbs.

A morphological evaluation of the tendons with ultrasonography was conducted by the same expert radiologist in both group with the aim of determine the quality of the repair in the surgical group and to detect the increasing tear size in the conservative group.

The study meets the ethical standards of Muscle, Ligaments and Tendons Journal 22.

\section{Statistical analysis}

A total sample size of 14 was considered adequate for overall comparison of the two groups assuming an a value of 0.05 (sensitivity of $95 \%$ ) and a $\beta$ value of 0.80 (study power, $80 \%$ ). The calculation of sample size was performed using $G^{\star}$ Power 3 software (Heinrich-Heine-University, Dusseldorf, Germany).

Differences at baseline between the 2 treatment groups were tested using the Mann-Whitney $U$ test. The Mann-Whitney $U$ test was used to assess differences in mean improvement from T0 to T2 in patients treated surgically or conservatively, from T0 to $\mathrm{T} 1$ 
and from $\mathrm{T} 1$ to $\mathrm{T} 2$ in conservative treatment group, as to assess differences at T2 between both treatments groups. The Chi-square test was used to evaluate differences in patient's satisfaction between the two groups.

All statistical analyses were performed by a single statistic using SPSS 20.0 software (IBM Corp, Armonk, NY, USA). Significance was defined at the 5\% level $(P<0.05)$.

\section{Results}

Baseline and characteristics are presented in Table I. Group A included 15 women and 5 men, with a mean age of 63.3 years $( \pm 4.7)$. The tear size was small in 9 patients, medium in 11 patients. The dominant limb was involved in 11 patients. The time from diagnosis to treatment was $2 \pm 1$ months.

Group B included 14 women and 6 men, with a mean age of 64.2 years (range \pm 3.8 ). The tear size was small in 8 patients, medium in 12 patients. The dominant limb was involved in 13 patients. The time from diagnosis to treatment was $1 \pm 0.2$ months.

At the latest follow-up, 2 patients out of group B had undergone surgical repair and were therefore excluded. When investigating the reason for this choice, both patients reported their symptoms were getting worse so they decided to switch to surgical repair. In group A, we registered a statistically significant improvement at T2 compared to T0 for the Constant score $(p=0.0035)$, the QuickDash $(p=0.0053)$ and the VAS scale $(p=0.01)$.

In group $B$, we registered a statistically significant improvement at T2 compared to T0 for the Constant score $(p=0.001)$, the QuickDash $(p=0.00052)$ and the VAS scale $(p=0.012)$. We also registered statistically and the VAS scale $(\mathrm{p}=0.048)$ (Tab.III).

The dynamometric evaluation showed, in group $A$ $93 \%$ of strength of involved versus uninvolved side, while in group B a $76,8 \%$ was detected with a statistically better performance in the surgically treated group $(p=0.0014)$.

Patient's satisfaction was excellent or good in $90 \%$ of group $A$ and in $60 \%$ of group B, while $10 \%$ of group A and $40 \%$ of group $B$ was unsatisfied or poorly satisfied $(p=0.036)$.

In the surgical group, the ultrasonographic evaluation showed a re-tear in 2 patients (10\%). In the conservative group, an increased tear size at the latest follow-up was found in $11 \%$ of patients as assessed with ultrasonography.

Out of group A, 15 patients (75\%) reported full compliance to the rehabilitation protocol, 2 patients (15\%) reported mean compliance, while 2 more patients $(10 \%)$ reported only poor compliance to the protocol proposed.

Out of group B, 14 patients (77.8\%) reported full compliance to the rehabilitation protocol, 2 patients $(11.1 \%)$ reported mean compliance, while 2 more patients $(11.1 \%)$ reported only poor compliance to the protocol proposed.

\section{Discussion}

The most important finding of this study is that a significant improvement was obtained in both treatments groups in patients with small to medium supraspinatus tears, although surgical repair yielded better results at the latest follow-up. Moreover, good to excellent results were achieved in $90 \%$ of patients in group A compared to $60 \%$ in group B. Thus, the hypothesis of the study was rejected. Another finding of the present study is that the analysis of strength measure-

Table I. Baseline characteristics.

\begin{tabular}{llll}
\hline Variables & Group A & Group B & p \\
\hline No. & 20 & 18 & \\
Sex, \% (number) female & $75(15)$ & $77(14)$ & 0.22 \\
Age, mean (SD) y & $63.3(4)$ & $64.2(3)$ & 1 \\
Follow-up, mean (SD) months & $18(2)$ & $18(3)$ & 0.77 \\
Constant, mean (SD) & $46.1(6)$ & $47.1(5)$ & 0.88 \\
Quick-DASH, mean (SD) & $46.2(3)$ & $47.4(4)$ & 1 \\
VAS, mean (SD) & $7(1)$ & $6.9(2)$ & 1 \\
\hline
\end{tabular}

better results for all the parameters evaluated at T1 compared to TO and slightly worse results, although not statistically significant, comparing results at $\mathrm{T} 2$ to those at T1 (Tab. II).

When comparing results of both groups at T2, statistical analysis showed better results in Group A for the Constant score $(p=0.004)$, the QuickDash $(p=0.012)$
Table II. Group B at the intermediate follow-up (T1).

\begin{tabular}{ll}
\hline Variables & Group B \\
\hline Constant, mean (SD) & $70.2(2.2)$ \\
Quick-DASH, mean (SD) & $19.5(1.5)$ \\
VAS, mean (SD) & $3.3(1)$ \\
\hline
\end{tabular}


Table III. Comparison between groups at the latest follow-up (T2).

\begin{tabular}{llll}
\hline Variables & Group A & Group B & P value \\
\hline Constant, mean (SD) & $80.2(3.4)$ & $68.55(5.2)$ & 0.004 \\
Quick-DASH, mean (SD) & $18.2(2.8)$ & $22.27(1.3)$ & 0.012 \\
VAS, mean (SD) & $2.1(1)$ & $4.4(2.2)$ & 0.048 \\
Dynamometric Evaluation (SD) & $93 \%(2)$ & $76,8 \%(5)$ & 0.001 \\
\hline
\end{tabular}

ment (s/s difference) showed significant better values in the surgical group compared to conservatively treated group. Moreover, the ultrasonographic examination at the latest follow-up (T2) showed comparable results between groups, with a re-tear rate of $10 \%$ in group $\mathrm{A}$ and a progression of tear size in $11 \%$ among patients of group B.

One of the strengths of the study is the strict selection of the patients which allowed an evaluation of two homogeneous groups, particularly regarding number and type of lesions. To our knowledge, very few report exist with those specific selection criteria, moreover considering that all the patients in the surgical group were treated by the same expert surgeon. However, this study has several limitations. First, the retrospective fashion with a relatively small number of patients enrolled and the short-term follow-up. Moreover, although we assessed the results of the repair and the tear size with imaging at the follow-up, we didn't perform any subgroup analysis to determine differences in successful repair compared to failures or how increasing tear in the conservative group could have had an impact on outcomes, if any. However, considering the small number of patients enrolled, we think that this subgroup analysis would not have had a clinical significance. Finally, although we performed a dynamometric assessment, this could not give us strength values as accurate as those measured by an isokinetic test. Moreover, we evaluate only abduction strength to rule out the status of the supraspinatus, while a complete assessment including elevation and both internal and external rotation could have helped to evaluate global shoulder function.

Moosmayer et al. ${ }^{23}$ in their randomized controlled study on 103 patients with 5 years of follow-up found better outcomes in patients operated on for a small to medium size RCT compared to those treated with physiotherapy plus secondary repair. In particular, they detected a mean difference of 5.3 points on the Constant score comparing both groups at the final follow-up, which is lower than 10.4 points considered necessary to detect clinically important differences ${ }^{24}$. However, when patients undergoing secondary repair were excluded, as was the case in our study, this difference reached 9.7 points, which is more similar to our results (11.6 points). Moreover, in $37 \%$ of tears treated with physiotherapy only, there was an increase in tear's size on ultrasound of $>5 \mathrm{~mm}$, associated with an inferior outcome.
Three other randomized controlled studies in literature report results which appear partially in conflict to those reported in this paper. The first is the report of Kukkonen et al. ${ }^{25}$ who showed no clinical advantage of surgery (acromioplasty alone or acromioplasty and rotator cuff repair) compared to conservative treatment at two years of follow-up, with all treatments achieving significant improvement with respect to preintervention time. Despite the similar short-term follow-up, this study included only very small supraspinatus tears (average size 12.5-14.8 mm), which, as suggested by biomechanical studies ${ }^{26}$, do not confer a biomechanical disadvantage to the shoulder, while larger tears that involve the entire supraspinatus may be the critical size that leads to tear progression and clinical worsening. The second report is that of Lambers Heerspink et al. ${ }^{27}$, who did not observe a difference in functional outcome 1 year after treatment, except for small significant differences in pain in favor of surgical treatment. However, this study included larger and multiple tendon tears with a less strictly selected group of patients compared to those enrolled in the present study. Moreover, they performed an open repair which didn't allow treatment of additional concomitant disorders, as long head biceps tendon pathology, reporting $73 \%$ repair failure rate. Those aspects should be highlighted when considering these results. The third is the report of Lee et al. ${ }^{28}$ who showed that effectiveness of the rehabilitation protocol is not inferior to arthroscopic repair for patients $>50$ years old with a less than medium-sized rotator cuff tear in a 1-year follow-up period. However, that study included either partialthickness or small to medium sized full thickness tears and the follow-up was only 1 year.

The dynamometric assessment showed better results in the surgical group with a $93 \%$ of strength of involved versus not involved side, which is consistent with the results of the repair and appears in line with values reported in literature ${ }^{29}$. Because of the small number of patients enrolled, we didn't evaluate the effect of re-rupture on strength measurements. However, as recently reported by Colliver et al. ${ }^{30}$ in their prospective study, no clear correlation seems to exist between strength recovery and early post-operative status of the tendons repaired. Moreover, although an isokinetic evaluation seems to be more appropriate, current clinical literature regarding rotator cuff tears displays significant variability in the types of strength results reported, despite being published in high-im- 
pact orthopedic journals ${ }^{31}$.

The ultrasonography imaging at follow-up showed a re-tear rate of $10 \%$ in group $A$, while a progression of tear size was found in $11 \%$ of group B patients. These results seem to be in line with those reported in the literature. Although it has been reported that most of re-ruptures happen during the first three months, and this seems to explain the number of suture failures observed in this study, on the contrary, one year of follow-up seems to be too short an interval to define the amount of progression of full-thickness $\mathrm{RCT}^{32}$.

Another finding of the present study is that we registered a worsening of all results in the conservative group between the intermediate (T1) and the final follow-up (T2), although it didn't reach significance. We could speculate that those results reflect the natural tendency of RCT to evolve with a progressive increase of tear size, tendon retraction and degenerative muscular changes ${ }^{28}$. Moreover, while during the first 12 weeks the rehabilitation protocol was performed with the help of a physiotherapist, after this period exercises were self-performed by patients. Although most of the patients $(77.8 \%)$ reported they were compliant with the protocol proposed, we are not able to assess the real compliance of the patients and if that could play a role in determining outcomes.

Considering the limitations of the study, future randomized controlled studies with more selective inclusion criteria and more standardized outcomes and strength value reporting are needed to consolidate this data.

In conclusion, at a short term follow-up, surgical treatment of small to medium supraspinatus tears yielded better clinical outcomes compared to conservative treatment, with better strength outcomes and a $10 \%$ of re-tear rate. Nevertheless, rehabilitation protocol still seems to offer acceptable results and could be a valuable option among patients not undergoing surgery.

Further studies, a larger sample of patients together with a better control of the patient's post-operative compliance are needed to determine if the rehabilitation protocol could be a valuable option for patients with small to medium size tear.

\section{Conflict of interest}

The Author has no financial or personal relationships with other people or organizations that could inappropriately influence their work.

\section{References}

1. Yamamoto A, Takagishi K, Osawa T, et al. Prevalence and risk factors of a rotator cuff tear in the general population. J Shoulder Elbow Surg. 2010;19(1):116-120.

2. Itoi $E$. Rotator cuff tear: physical examination and conservative treatment, J Orthop Sci. 2013;18:197-204.

3. Codman EA. Complete rupture of the supraspinatus tendon. Operative treatment with report of two successful cases. 1911.
J Shoulder Elbow Surg. 2011;20(3):347-349.

4. Arntz CT, Matsen FA 3rd, Jackins S. Surgical management of complex irreparable rotator cuff deficiency. J Arthroplasty. 1991;6:363-370.

5. Galatz LM, Ball CM, Teefey SA, Middleton WD, Yamaguchi K. The outcome and repair integrity of completely arthroscopically repaired large and massive rotator cuff tears. J Bone Joint Surg Am. 2004;86:219-224 .

6. Gerber C. Latissimus dorsi transfer for the treatment of irreparable tears of the rotator cuff. Clin Orthop Relat Res. 1992;275:152-160.

7. Karas SE, Giachello TL. Subscapularis transfer for reconstruction of massive tears of the rotator cuff. J Bone Joint Surg Am. 1996;78:239-245.

8. Rockwood CA Jr, Williams GR Jr, Burkhead WZ Jr. Debridement of degenerative, irreparable lesions of the rotator cuff. $J$ Bone Joint Surg Am. 1995;77:857-866.

9. Longo UG, Franceschi F, Berton A, Maffulli N, Droena V. Conservative treatment and rotator cuff tear progression. Med Sport Sci. 2012;57:90-99.

10. Kijima H, Minagawa H, Nishi T, Kikuchi K, Shimada Y. Longterm follow-up of cases of rotator cuff tear treated conservatively. J Shoulder Elbow Surg. 2012;21:491-494.

11. Kuhn JE, Dunn WR, Sanders R, et al. Effectiveness of physical therapy in treating atraumatic full-thickness rotator cuff tears: a multicenter prospective cohort study. J Shoulder Elbow Surg. 2013;22(10):1371-1379.

12. Oliva F, Piccirilli E, Bossa M, et al. ISMuLT - Rotator Cuff Tears Guidelines. Muscles Ligaments Tendons J. 2016;13;5(4):227263.

13. Koubaa S, Ben Salah FZ, Lebib S, Miri I, Ghorbel S, Dziri C. Conservative management of full-thickness rotator cuff tears: a prospective study of 24 patients. Ann Readapt Med Phys. 2006;49:62-67.

14. Itoi E, Tabata S. Conservative treatment of rotator cuff tears. Clin Orthop Relat Res. 1992;(275):165-173.

15. Kukkonen J, Joukainen A, Lehtinen J, Mattila KT, Tuom- inen EK, Kauko T, et al. Treatment of non-traumatic rota- tor cuff tears: a randomised controlled trial with one-year clinical results. Bone Joint J. 2014;96B:75-81.

16. Cofield RH. Subscapular muscle transposition for re- pair of chronic rotator cuff tears. Surg Gynecol Obstet. 1982;154:667672.

17. De Carli A, Vadalà A, Zanzotto $E$, et al. Reparable rotator cuff tears with concomitant long-head biceps lesions: tenotomy or tenotomy/tenodesis? Knee Surg Sports Traumatol Arthrosc. 2012;20(12):2553-2558.

18. Montanari G, Facilitazioni neurocinetiche progressive. Elaborazione del concetto kabat. Editor: Edi Ermes. 2004.

19. Constant $\mathrm{CR}$, Murley AH. A clinical method of functional assessment of the shoulder. Clin Orthop Relat Res. 1987;(214): 160-164.

20. Gummesson C, Ward M, Atroshi I, The shortened disabilities of the arm, shoulder and hand questionnaire (QuickDASH): validity and reliability based on responses within the full-length DASH. BMC Muscoloskelet Disord. 2006;7:44.

21. McCormack HM, Horne DJ, Sheather S. Clinical applications of visual analogue scales: a critical review. Psychol Med. 1988;18(4):1007-1019.

22. Padulo J, Oliva F, Frizziero A, Maffulli N. Muscles, Ligaments and Tendons Journal - Basic principles and recommendations in clinical and field science research: 2016 update. MLTJ. 2016;6(1):1-5.

23. Moosmayer S, Lund G, Seljom US, et al. Tendon repair compared with physiotherapy in the treatment of rotator cuff tears: a randomized controlled study in 103 cases with a five-year follow-up. J Bone Joint Surg Am. 2014;96(18):1504-1514.

24. Kukkonen J, Kauko T, Vahlberg T, Joukainen A, Aarimaa V. 
Investigating minimal clinically important difference for Constant score in patients undergoing rotator cuff surgery. J Shoulder Elbow Surg. 2013;22:1650-1655.

25. Kukkonen J, Joukainen A, Lehtinen J, et al. Treatment of Nontraumatic Rotator Cuff Tears: A Randomized Controlled Trial with Two Years of Clinical and Imaging Follow-up. J Bone Joint Surg Am. 2015;97(21):1729-1737.

26. Oh JH, Jun BJ, McGarry MH, Lee TQ. Does a critical rotator cuff tear stage exist? A biomechanical study of rotator cuff tear progression in human cadaver shoulders. J Bone Joint Surg Am. 2011;93(22):2100-2109.

27. Lambers Heerspink FO, van Raay JJ, Koorevaar RC, et al. Comparing surgical repair with conservative treatment for degenerative rotator cuff tears: a randomized controlled trial. J Shoulder Elbow Surg. 2015;24(8):1274-1281.

28. Lee WH, et al. Clinical Outcomes of Conservative Treatment and Arthroscopic Repair of Rotator Cuff Tears: A Retrospective Observational Study. Ann Rehabil Med. 2016;40(2):25262.
29. Visonà $E$, Paladini $P$, Merolla G, Cerciello S, Porcellini G. Strength recovery after arthroscopic anterosuperior cuff repair: analysis of a consecutive series. Musculoskelet Surg. 2015; 99;Suppl1:S37-42.

30. Colliver J, Wang A, Joss B, et al. Early postoperative repair status after rotator cuff repair cannot be accurately classified using questionnaires of patient function and isokinetic strength evaluation. J Shoulder Elbow Surg. 2015. Makhni EC, Steinhaus ME, Morrow ZS, et al. Outcomes assessment in rotator cuff pathology: what are we measuring? J Shoulder Elbow Surg. 2015;24(12):2008-2015.

31. Nho SJ, Shindle MK, Adler RS, Warren RF, Altchek DW, MacGillivray JD. Prospective analysis of arthroscopic rotator cuff repair: subgroup analysis. J Shoulder Elbow Surg. 2009; 18:697-704.

32. Goutallier D, Postel JM, Bernageau J, Lavau L, Voisin MC Fatty muscle degeneration in cuff ruptures. Pre- and postoperative evaluation by CT scan. Clin Orthop Relat Res. 1994; (304):78-83. 